\title{
Counter-Strategy to Emergencies in the Coal Mining Industry Based on the Use of Multi-Agent Systems and Fuzzy Logic
}

\author{
Albert Bakhtizin ${ }^{1}$, Evgeniy Loginov ${ }^{2}$, Alexander Shkuta $^{3}$, and Vladimir Abramov ${ }^{1}$ \\ ${ }^{1}$ Central Economic and Mathematics Institute, Russian Academy of Sciences, 117418 Nakhimovsky \\ Prospect 47, Moscow, Russia, Moscow, Russian Federation \\ ${ }^{2}$ Market Economy Institute, Russian Academy of Sciences, 117418 Nakhimovsky Prospect 47, \\ Moscow, Russia, Moscow, Russian Federation \\ ${ }^{3}$ Financial University under the Government of the Russian Federation, 125993 Leningradsky \\ Prospekt 49, Russian Federation, Moscow
}

\begin{abstract}
Research carried out at the expense of the grant RFBR № 17-0200416-OGN is devoted to the consideration of problems of formation of a qualitatively new model of monitoring, identification and forecasting of any forms of threats and risks in the field of security of coal mining facilities. Research take into account their new aspects (cross-border network structures, lack of a single control center, presence of a large number of quasi-autonomous organizational and technical elements with their own management trajectories and localized interests etc.). Monitoring and modelling these processes is the basis of the systemic strategies of prevention of emergencies. Developing specific measures for information assurance organizational process in implementation of management action is based on the convergence of information, telecommunication and computing services to government agencies as a control object with a significant number of deviant implemented operational variables with the absence or delay of information signals about their dynamics and ensuring interdepartmental cooperation.
\end{abstract}

\section{Introduction}

Taking into account new aspects research aims in improving the efficiency of Russian mechanisms for prevention and elimination of emergencies because of critical deficit of information about threats and risks in the field of safety of coal mining facilities - through monitoring and modeling using multi-agent systems and fuzzy logic. Authors propose to integrate into a single complex of organizational structures and information systems of various government agencies as multi-purpose agents formalized as a network-centric information grid of government in the field of emergencies of Russia and EAEU in the future as well.

Prevention and liquidation of emergencies requires very difficult organizational work. It is necessary to develop basic technologies and systems of monitoring, gathering, analyzing, modeling, predicting, identifying threats, as well as supporting the development of management decisions, planning of countermeasures, their implementation, support, 
Iеeaback and measures to improve processes and proceaures. is aiso requirea to aeveiop emergencies response systems themselves as a highly dynamic set of nonlinear operational processes that are difficult or impossible to collect information proactively.

In recent years developments in the use of new information technologies for the prevention and elimination of emergencies use information systems for civil, military and special purposes have been actively developing in Russia [1-10].

Despite the active development in this area, there is a certain lag of ideas on the problem of ensuring the safety of coal mining facilities in relation to qualitatively new objects of the organizational environment. There is a specific kind of initiated loss of control of coal mining facilities due to the increase of intelligent devices with a high degree of autonomy of behavior (smart grid, etc.), as the classical mono-agent objects, which has not yet found a sufficiently complete reflection in scientific research and practical development of relevant government agencies and services.

Means and methods of destructive impact on coal industry can be fundamentally new. Emergencies contribute to rapid growth of facilities with a large number of automated services that do not have a common regulatory center. At the same time, a number of objects of interconnected coal mining industry may be outside the scope of their possible regulation and there is not enough information about their activities. Monitoring and modeling of these processes requires the use of agent-based models and fuzzy logic methods to overcome critical deficit of information about the nature of emergency threats and risks.

It is necessary to improve the directions and methods of functioning mechanisms of prevention and elimination emergencies with the use of multi-agent systems. Efficiency of various state agencies can be analyzed as a kind of agents with a significant number of deviating operating variables implemented with the absence or delay of information signals about their related dynamics.

The most important element of such a mechanism is the information support of the organizational process of implementation of management actions by using new information technologies. These technologies should enable the practical implementation of an integrated and systematic approach to the problem of countering difficult-to-predict phenomena in the context of critical shortage of information on threats and risks of emergency nature.

Under these conditions, it is necessary to develop approaches to monitoring and modeling of processes of counteraction to nonlinear processes of emergencies. Multi-agent systems and fuzzy logic take into account the inability to provide high observability of the processes of functioning of a large number of geographically dispersed objects of the coal mining industry without significant changes in information management systems.

It is necessary to improve organizational cooperation by integrating organizational structures and information systems of various government agencies as multi-purpose agents into a single complex. Such a system can be formalized as a network information grid of government in the field of emergency response.

\section{Materials and Methods}

To improve the efficiency of management mechanisms as a system of measures aimed to ensure the safety of objects of the coal mining industry in Russia it is necessary to highlight the following.

1. Analysis of processes and patterns in relation to the dynamic totality of interaction of different categories of objects. Such analysis allocate the basis of direct or indirect participation in coal mining industry in Russia including collecting information about patterns and trends of processes of functioning of distributed information systems.

2. Analysis of peculiarities of functioning of the Russian system of prevention of emergencies in General and its separate institutional mechanisms for the development of proposals for its 
systemic, integrated development. This will improve the interaction between the various Russian agencies, definition of ways of formation of network-centric information management system (network-centric information grid in the sphere of prevention of emergencies) integrating the segments of information and telecommunication infrastructure of government administration, rescue and other organs and services.

3. Development of a management model for monitoring and control based on the gathering and analysis of large amounts of unrelated information in various databases and global information networks for coordination at the interdepartmental level on information technology chains of information collection, processing, store and exchange of information within government and special agencies.

4. Development of mathematical support for the implementation of a new approach to the assessment of the initial factors and support management decision-making in the field of monitoring, identification and prevention of any forms of threats and risks. Security of coal mining facilities can base on the use of multi-agent systems and fuzzy logic methods for these purposes.

5. Analysis of the problems of overcoming the existing limitations for the development of monitoring, identification and forecasting of any forms of threats and risks in the sphere of safety of objects of the coal mining industry. Including computer simulations with agentbased techniques simulate the information interactions helps identify a wide range of events that may be relevant to emergencies as highly dynamic aggregate nonlinear operational processes. It is difficult or impossible to collect information about them in a pre-emptive way using the multi-aspect systematics of the semantics of the analyzed connections.

6. Development of the method of practical design of the information technology complex for monitoring, identification and forecasting of any forms of threats and risks in the field of security of coal mining facilities and methods of identification of the chronotype, participants, role structure and resource base of emergency response. The method can be based both on the analysis of a single event, and on the aggregation and comparison of diverse events and network flows of such operations.

7. Identifying measures to improve the efficiency of institutional mechanisms of prevention of emergencies through the reconfiguration of the volumes, structure and conditions of the various state departments. The transition from fuzzy pins to the exact value of the controlled parameter with the formation of a dynamically adaptable to the various state agencies set of tools support the process of implementation of control and monitoring, informationanalytical and organizational-administrative tasks, dealing with emergencies within the framework of network-centric information grid in the sphere of emergencies prevention.

\section{Results}

The principle of effective organizational interaction in this mechanism is to integrate them into a single network of organizational structures and information systems. Various government agencies can cooperate with help of multi-agent systems in the framework of their current activities with a view to immediate blocking of the upsurge of emergencies due to weather phenomena or other unpredictable reasons.

To do this, it is necessary to clarify the possibility of integration of informationcomputing platforms at the interregional Russian and Eurasian levels to expand the possibilities of identifying a wide range of events that may be relevant to emergencies as a highly dynamic set of nonlinear operational processes. It is necessary to analyze the current conditions for the transition to the creation of a network-centric information grid of emergency response. Principles of convergence of information, telecommunications and computing services makes it possible to cooperate various government agencies as objects of 
management, witn a signincant number or deviaung operaung variabies impiemented witn the absence or delay of information signals about their dynamics.

An important stage in the implementation of the above actions is the formation of a planning mechanism through the implementation of recovery, rescue or other operations in respect of the most dangerous forms of emergency. Transition from fuzzy conclusions to the exact value of the regulated parameter in the key areas of the functional activities of a particular state agency can reallocate key operating nodes to improve the sustainability and adequacy of the management model in relation to the organization of interdepartmental actions of various government agencies.

Within the EAEU, it is necessary:

- to outline the ways of development in interdepartmental cooperation as a multi-agent system based on the system of aggregated information centers of state agencies within the network information grid of emergency response;

- to identify the areas of introduction of new information, telecommunication and computing services to expand the analyzed field of events that may be relevant to emergency situations with a large number of unpredictable management behavior using a multi-aspect systematics in the semantics of analyzed relationships;

- to determine the aspects of solving the problem of reconfiguration the volume, structure and conditions of actions of various government agencies.

Within the framework of the Russian government and special agencies, it is necessary: - to form the directions in development of information and analytical mechanisms using intelligent technologies of analysis and data processing within the network-centric information grid of emergency response in different scenarios of emergency dynamics in relation to their most dangerous forms;

- to determine the direction of expanding control of total data sets available for analysis in databases and global information networks in the context of external information unfriendly to the Russian authorities of companies from political and economic sanctions;

- to identify within the EAEU main blocks of interdependence between government and special agencies from each other including the strategic configuration of a promising system in organization of various state agencies interdepartmental actions with a cooperative structure of management and special measures for the prevention and elimination of emergencies;

- to substantiate the directions of designing a set of tools to support the implementation of control and monitoring tasks of emergency response with their approximation to the system of world standards of monitoring and control. Such a set will include the activities of government and special agencies in the modelling framework for the implementation of an effective way to prevent and eliminate emergencies in coal mining industry of Russia and in the future with the extension to the member States of the Eurasian economic Union.

\section{Conclusion}

Emergencies prevention requires the formation of information-telecommunication environment of the new generation with the introduction of the interagency converged computing platform. Creating a control center can provide inter-regional and international information network for the implementation of international monitoring and coordination of all forms of threats and risks in the sphere of coal mining objects safety with the expansion of the common information field of data available for analysis in the specific territorial area of the EAEU.

Thus, the implementation such measures should develop a concept of new mechanisms for monitoring, identification and forecasting any forms of threats and risks in the field of security of the coal mining industry in Russia and other EAEU members. It is proposed to 
optimize the cooperation of state agencies in the framework of the network-centric information grid of emergency response, which allows forming mechanisms and integrating the functions of management to control and distribute information in a single system.

\section{References}

1. A.R. Bakhtizin, E.M. Bukhvald, A. Kolchugina, Advances in Systems Science and Applications, 16(3), 11-20 (2016)

2. S.M. Bandini, S. Manzoni, G. Vizzari, Journal of Artificial Societies and Social Simulation, 12(4), 32-47 (2009)

3. E. Bonabeau, Proc. National Academy of Sciences, 99(3), 134-142 (2011)

4. Deguchi H. Economics as an Agent-Based Complex System (Springer, Berlin, 2004)

5. W. H. Hamilton, The Institutional Approach to Economic Theory. American Alternatives to Economic Orthodoxy: A Reader in Political Economy (NYAED, New York, 1987)

6. E.L. Loginov, A.N. Raikov, Thermal Engineering, 62(4), 233-239 (2015)

7. M. Oremland, Journal of Artificial Societies and Social Simulation, 17(2), 1-6 (2014)

8. J. Von Neumann, Theory of Self-reproducing Automata (Urbana, London, 1966)

9. M. Wooldridge, An Introduction to MultiAgent Systems (John Wiley \& Sons Ltd, London, 2002)

10. M. J. Woolridge, N. R. Jennings, The Knowledge Engineering Review, 10(2), 115-152 (1995) 